\title{
The Role of Clinical Hypnosis \& VR in Special Education
}

\author{
https://doi.org/10.3991/ijes.v9i4.26147 \\ Athanasios Drigas $^{1(凶)}$, Eleni Mitsea $^{1,2}$, Charalabos Skianis ${ }^{2}$ \\ ${ }^{1}$ N.C.S.R. 'Demokritos' Athens, Agia Paraskevi, Greece \\ ${ }^{2}$ University of the Aegean and Communication Systems Engineering \\ Department, Samos, Greece \\ dreit.demokritos.gr
}

\begin{abstract}
Clinical hypnosis is becoming increasingly recognized as a valuable clinical intervention for a wide range of psychological and medical problems. The current review paper seeks to determine the role of clinical hypnosis in learning difficulties, emotional or behavioral problems and physical disabilities. In addition, we co-examine the effectiveness of virtual/augmented reality hypnosis. Finally, we discuss the possible uses of clinical hypnosis within the educational settings. The results showed that hypnosis may hold the keys for new types of metacognitive strategies based on non-verbal pathways of effortless self-regulation. We are on the eve of a new philosophy in special education, open to ground-breaking intervention strategies that recognize the fundamental role of subconsciousness in the treatment of learning and other disabilities. Clinical hypnosis seems to be a promising strategy with high potential to be applied in the educational settings.
\end{abstract}

Keywords — clinical hypnosis, virtual/augmented reality hypnosis, special education, metacognition, effortless self-regulation

\section{Introduction}

Millions of people, mainly children and adolescents, are diagnosed with learning and other disabilities, while the recommended medications often are often proved to be insufficient. Scientists seek non-pharmacological solutions in order to deal with the arising challenges with the aim of ensuring symptoms elimination, psychological well-being and achievement in every strata of life [1]. Mind-Body interventions constantly gain ground as an adjunct tool to conventional medical care. Clinical hypnosis is considered a mainstream mind-body modality for the treatment of a variety of psychological and medical problems [2-3].

Although there is a confusion regarding the "real" meaning of hypnosis, the latest definition reports: "Hypnosis is a state of consciousness involving inwardly focused attention and reduced peripheral awareness characterized by an enhanced capacity for 
response to suggestion" [4]. Hypnosis involves, at least, two components: hypnotic induction and suggestions. An experienced and specialized professional give patients self-directed therapeutic suggestions to induce profound alterations in sensations, perceptions, thoughts and behaviors [2,4-5]. In contrast to other interventions, clinical hypnosis allows individuals to override deeply entrenched processes quickly and effortlessly [5].

Clinical hypnosis is already established in various clinical applications, especially pain management and other medical conditions. It has already been recommended as an effective intervention strategy for managing pain, obesity, smoking cessation. In medicine, there is acceptable evidence in treating gastrointestinal disorders, asthma, diabetes, hypertension, immunological problems, dermatological diseases [3].

Hypnosis is considered as an effective modality not only for adults, but also for children and adolescents. Young people are thought to be more hypnotically suggestible than adults. However, research regarding the efficacy of hypnosis to younger candidates is lacking or is still at an early stage of development [2-3]. Although extensive research has been carried out on clinical hypnosis in various medical conditions, far too little attention has been paid to clinical hypnosis as an intervention strategy for people with learning and other disabilities. The aim of the current review is to survey the contemporary literature to determine the role of clinical hypnosis in treating people with learning difficulties, emotional or behavioral problems or physical disabilities.

Over the last few years, researchers and health care teams explore the role of technology to enhance the effectiveness of traditional and non-pharmacological interventions. Techniques like hypnosis and virtual reality are being investigated in numerous studies providing considerable opportunities [6]. In addition, we examine the impact of Virtual/ Augmented reality in hypnosis with a special focus on treating disabled people.

\section{Clinical hypnosis for people with special needs \& learning disabilities}

\subsection{Clinical hypnosis for attention deficit hyperactivity disorder (ADHD)}

In a randomized controlled trial, Hiltunen et al. [7] investigated the effectiveness of hypnosis in treating adults with ADHD. The participants were randomized to three treatment groups (hypnotherapy, CBT, cognitive training) and to a control group. The themes of the hypnosis sessions included hypnotic induction and suggestions promoting stillness, motivation to change, attention, initiation of activities, memory, self-esteem, and management of fears, impulsivity and anger. Report questions and independent evaluations were used as outcome measures. The participants were evaluated after three and six months from the end of the treatment. The results showed that hypnosis reduced the symptoms of ADHD to a greater extent than CBT especially in the area of emotional regulation. The participants were more attentive with positive thoughts and beliefs, reduced hyperactivity, and impulsivity symptoms. At follow-up, in the intervention group the outcomes remained stronger and more stable. The authors concluded that clinical hypnosis has a positive and more stable impact on attentional, emotional and other executive functions. 
Virta et al. [8] examined the impact of hypnosis and hypnotic suggestions on the symptoms of ADHD with a special focus on attention processes. Twenty-seven adults with ADHD participated in the experimental group and thirty-one volunteers in the control group. The researchers administered the neuropsychological task "Continuous Performance Test" to measure participants' sustained and selective attention in four different conditions: (1) before hypnosis, (2) after a hypnotic induction, (3) after hypnotic suggestions about speed and accuracy, and (4) after the termination of hypnosis. During the third condition of hypnotic suggestions, the participants were instructed to be focused, accurate and quick, to avoid distractions, to resist impulses. The results showed that there was a statistically significant decrease in reaction times in both ADHD and control group after hypnotic suggestions. The study concluded that hypnotic suggestion has an important impact on reaction times in the sustained attention task.

\subsection{Clinical hypnosis for autism spectrum disorder (ASD)}

According to Sugarman et al. [9] hypnosis combined with biofeedback can be utilized to develop self-regulation habits and adaptive behaviors for people with ASD. Specifically, they describe hypnotic strategies that utilize restrictive repetitive behaviors in trance as the resources for comfort and control. The hypnotist guides patients to virtually experience situations in which they have engaged in restrictive repetitive behaviors and transform them to effective self-regulation behaviors. At the same time, biofeedback regulates the autonomic nervous system, encouraging lower sympathetic tone and arousal. The authors conclude that hypnosis in combination with biofeedback allow a person with ASD to make improvements in eyes contact, emotional receptivity and comprehension, motor behaviors and social engagement.

Gardner et al. [10] described the use of an adjunctive hypnotherapy in a 16-year-old boy with autism possessing an amazing music memory. Anticipating that the boy's musical skills might boost his involvement in hypnotherapy, the patient was suggested to compose two short musical themes representing frustration in $\mathrm{C}$ minor scale and happiness in $\mathrm{C}$ major scale. Instead of verbal instructions and suggestions, the hypnotherapist asked patient to listen to the two compositions and suggested to recall the first theme to replace the biting behavior and recall the second theme to strengthen feelings of confidence. To penetrate in deeper hypnotic states, the hypnotherapist used the boy's favorite religious musical piece as a hypnotic induction. Surprisingly, the patient responded with eye closure, spontaneous hand catalepsy indicating a deep trance. Overall, the patient changed unacceptable habits with more acceptable behaviors. He stated that every time he was frustrated, he recalled the music, so he did not bite his fingers. He began to observe and recognize frustrating experiences with clarity, to make hypothesis about the feelings of others, to express humor in his waking discusses. He gained confidence in his ability to self-control and generalized the hypnotic behavior to the waking state.

Overall, these results indicate that hypnosis may be a therapeutic modality in children diagnosed with autism especially when therapists utilize patients' obsessions as strength on which to base therapeutic strategy. However more research on this topic needs to be undertaken. 


\subsection{Clinical hypnosis for dyslexia/dyscalculia}

Visual-spatial abilities play a pivotal role in learning disabilities. Reading and mathematics difficulties, for instance, are inextricably linked to attentional and visual-spatial deficits. Giovagnoli et al. [11] found that young children with dyslexia perform worse in tasks requiring spatial attention span, visual-object recognition to perceive the global form of a figure. Developmental dyscalculia is thought to be a specific impairment of mathematics ability.

Szucs et al. [12] used 16 tests and nine experiments in order to identify the dominant features of dyscalculia selecting participants from a pool of 1.004 school children. They found that the dominant features of developmental dyscalculia are visuospatial working memory, visuospatial short-term memory, and inhibitory function (interference suppression) impairment.

Incognito et al. [13] investigated possible hypnotizability-related differences in the ability of spatial imagery. Two hundred and fifty university students were submitted to a test of mental rotation which is one of the most used measures of spatial ability, since it demands generation and storage of visuo-spatial mental images. The results showed that highly hypnotizable individuals performed the tests better. These finding further support the idea that hypnosis could enhance visuo-spatial impairments.

Lindeløv et al. [14], in a randomized controlled trial, examined whether hypnotic suggestions can improve working memory functions. They recruited participants who had sustained acquired brain injury. The experimental group received hypnotic suggestions about enhancing working memory function including age regression and visualizations of brain plasticity. Participants were tested on working memory tests. The results showed that targeted hypnotic suggestions have a positive and long-lasting effect on working memory performance. The authors assumed that hypnotic suggestions elicited mental imagery which probably improved working memory capacity. They also claimed that working memory deficits are not irreversible and the hypnotic suggestions act as retrieval clues for existing but non-used strategies.

\subsection{Clinical hypnosis for sensory and physical impairments}

Muller et al. [15] conducted an fMRI study to identify the brain regions involved in motor imagery as compared with real motor performance after a hypnotic trance. The findings showed that the brain areas activated during hypnosis imagination of a simple motor task are in a kind of "linking" position between motor imagery, motor planning and executing. The activated areas represent central nodes of the salient network which integrates sensory, emotional and cognitive information and contributes to a variety of functions including communication, social behavior and self-awareness. Most importantly, the data showed that there is prominent activation of the thalamus, solely during motor imagery. Thalamus is considered the "gate to awareness", participates in sensory control processes and it has a special role in attention. Hypnotic trance is likely to activate the critical node in the motor path from basal ganglia to higher motor areas responsible for motor imagery. In addition, the study also substantiates that the activated brain regions are involved in implicit learning.

Gravitz et al. [16] examined the effectiveness of non-verbal hypnotic techniques across several sensory dimensions for helping a brain-damaged centrally deaf 
36-year-old female patient. The hypnotic techniques included optical fixation on the therapist's hand, vibration stimuli, light shoulders pressure, and arm stroking. With hypnosis, the patient's physical and emotional behavior was improved. In addition, she reported that she felt more relaxed, able to control pain and movement. She also slept at night for longer. Fifteen months later, she also mentioned that her social life had been improved. Finally, the therapist observed that her speech was better understood.

Flamand-Roze et al. [17] conducted a review of the literature from 1984 to 2015 regarding the role of hypnosis in treating movement disorders. The authors found that hypnosis possibly alleviates motor deficiencies such as tics associated with Gilles de la Tourette syndrome especially when combined with physical training. They argued that hypnosis can act indirectly on movement disorders by improving sleep quality, anxiety, mood and self-esteem. However, there is evidence that hypnosis activates brain areas involved in motor control processes.

\subsection{Clinical hypnosis for down syndrome}

Garitte et al. [18] investigated whether children with Down's Syndrome are capable of being hypnotized and the cognitive variables that can mediate the hypnotic response. The sample included twelve participants, seven girls and five boys, ages ranging from six to seventeen. The researchers assessed the following cognitive variables namely vocabulary comprehension, perception, mnemonic recuperation, and abstraction. Results showed that the children with Down Syndrome were able to respond to hypnotic suggestions, both motor and cognitive. In addition, a strong correlation was found between hypnotic response and the cognitive variables with prominent the memory recuperation. The findings highlight that the children with Down Syndrome are receptive to hypnosis, so they could benefit from its therapeutic effects. In addition, hypnosis activates those channels and skills that are more developed in children with Down Syndrome such as visual encoding of information, comprehension and adaptation. Finally, hypnosis seems to improve the retrieval of information, a necessary process in learning and intelligence improvement.

\subsection{Clinical hypnosis for oppositional defiant disorder}

Iglesias et al. [19] examined the effectiveness of hypnosis in a case of preschool age child with oppositional defiant disorder with concomitant emotional dysregulation and secondary behavioral disruptiveness. The hypnotic procedure took the form of a game. The results showed a significant reduction of the out-of-control episodes. The child gained the ability to monitor, control and change emotional reactions. The positive gains remained at the follow-up test six months later.

\subsection{Clinical hypnosis for language disorders}

Kaya et al. [20] conducted an innovative preliminary study investigating the combined effect of hypnosis and diaphragmatic as well as respiratory exercises in the treatment of stuttering. Fifty-nine stutterers participated in an eight-session program which involved the following hypnotic procedures: hypnotic induction, hypo- and 
hyper-amnesic suggestions, eye and body catalepsy, past and forward projections and consolidation suggestions and post-hypnotic suggestions. During hypnotherapy session, the participants took part in a series of fluency exercises repeating alphabet, strings of syllables, telling stories, repeating polysyllabic words. After sessions, the participants practiced abdominal weightlifting to strengthen their respiratory muscles and the diaphragmatic movement. The results showed significant differences between pre- and post-treatment measures. In addition, 55 of the 59 clients reported that they felt relaxed and empowered and their improvement maintained. Combining deep hypnotic trance with abdominal weightlifting, the patients exercised muscles needed for the regulation of speech, relaxed, and were reprogrammed with the positive belief that their mind and body are "intelligent" enough to overcome dysfunctions.

Sehan et al. [21] examined the effects of light trance post-hypnotic suggestions to reading comprehension. University students with low reading comprehension scores $(32 \%)$ were instructed to relax during reading task, be more focused, positive, and motivated. After hypnosis, the students improved their ability to comprehend (93\%). In the unstructured interview, the students reported that they felt more focused, less distracted with a better ability to recall the content of the text. They also stated also that they were more engaged to the text, and everything seemed clearly connected.

Wark [22] reviewed the literature to investigate the effectiveness of alert hypnosis in comparison with traditional techniques in complex cognitive abilities such as reading comprehension. It was found that alert hypnosis is associated with improvements in reading speed, listening, and reading comprehension.

\subsection{Clinical hypnosis for giftedness}

Bowers et al. [23] explored the relationship between hypnotizability and creativity. Their review supports the idea that hypnosis boosts superior creativity in writing and problem solving since it possibly activates human's ability to associate without interference of volition selection strategies. Gur et al. [24] divided thirty-six highly susceptible subjects into hypnosis, simulation and walking groups. Afterwards, the participants were given a test that measures creativity. It was found that the hypnotized group scored higher than all control groups especially on non-verbal creativity (the ability to think creatively with pictures). Together, these studies outline that the use of hypnosis can enhance creativity, a prerequisite for developing higher order abilities.

Flow state refers to a state of heightened but effortless attention and concentration in a task to achieve peak performance. Bowers et al. [25] explored the relationship between hypnosis and flow state using a sample of 179 college students. The data showed that hypnosis evokes flow-like experiences. Pates et al. [26] examined the effects of a hypnosis intervention on flow states and high-performance sport. Four competitive female players were introduced to the training program composed of hypnotic induction, hypnotic regression, and trigger control. The researcher utilized Ericksonian hypnosis throughout the induction and the staircase technique in the trance stage. The findings indicated that three of the four participants increased their mean flow scores. The players mentioned that they felt more determined, focused, and happy. Thus, hypnosis could be used to develop higher frequency flow states assuring the factors that 
must be present for optimal performance namely intrinsic motivation and reward, loss of self-consciousness, action awareness, enjoyment, lucidity, challenge-skills balance.

Mossbridge et al. [27] examined the idea that hypnosis could help people manifest extraordinary abilities such as the ability to feel unconditional love, to predict events, to observe and intentionally influence the probability of random events. Fifty-four individuals participated in a crossover design. Before and after receiving the hypnotic suggestions by an experienced hypnotherapist, each participant was given the same set of online self-reports that measure supernormal performance, unconditional love and micro-psychokinesis. Hypnotic suggestions led to a significant and lasting increase in the self-reported feeling of unconditional love, a self-transcendent state in which love for oneself and others freely felt without expectations for return. In addition, the researchers found that participants had better performance on precognition under the unconditional love suggestion. Additional research is required. However, it seems that hypnosis may provoke the emergence of hidden and superior abilities such as unconditional love allowing freedom from the constraints of perception.

\subsection{Clinical hypnosis for anxiety disorders}

Valentine et al. [28] conducted a meta-analysis of the literature investigating the effectiveness of hypnosis in treating anxiety. The authors examined 15 studies incorporating 17 trials and found that hypnosis reduced anxiety more than about $84 \%$ of control participants. The authors concluded that hypnosis is a highly effective intervention and possible more effective than other common interventions.

In a single case study, Byron et al. [29] explored whether hypnosis could help a 15 -year-old anxious student with a social and communication disorder and inability to attend school. After hypnosis intervention, there was a reduction in anxiety, while, at the same time, strengthening of self-confidence, social communication and school attendance were observed. The student improved his emotional well-being and social inclusion. Authors concluded that hypnosis could be used as an adjunct to educational psychology for anxiety management, strengthening teaching in the specific area of social communication.

Halsband et al. [30] investigated the effects of a brief hypnosis on the fear processing structures of the brain in participants with phobia. Using functional magnetic resonance imaging (fMRI), the researchers observed the brain activity changes of twelve dental phobic and 12 healthy controls during a fear condition and after a brief hypnotic intervention. While in the fear condition, the phobic's group showed increased activation in the anxiety circuit namely amygdala, anterior cingulate cortex, insula and hippocampus, during hypnosis they showed a significantly reduced activation in all of these areas. These findings are of great importance considering that amygdala plays an important role in the unconscious processing of phobic threats and learned weaknesses. Insula is co-activated with amygdala during emotional processing, while hippocampus is involved also in the recollection of unpleasant memories. Finally, anterior cingulate cortex participates in goal directed behavior and conscious regulation. Thus, it is obvious that hypnosis can be a powerful tool for inhibiting the reaction of fear. 


\subsection{Clinical hypnosis for depression}

Alladin et al. [31] investigated the effectiveness of a 16-week clinical hypnosis treatment in depressive symptoms. Eighty-four chronic depressive patients were randomly assigned to either clinical hypnosis or cognitive-behavioral therapy group. The hypnotic procedures included hypnotic induction, ego-strengthening suggestions, expansion of awareness and positive mood induction. There was significantly larger decrease in depression, anxiety and hopelessness for clinical hypnosis intervention. In addition, 6-month and 12-month follow ups revealed maintenance of the effect size. According to the authors, clinical hypnosis aimed at unconscious restructuring, by weakening depressive pathways developed through negative self-focusing and strengthening happy pathways of positive imagery. The patients disengaged from negative self-hypnosis, namely rumination and distorted memories, so they felt self-empowered.

Fuhr et al. [32] conducted a monocentric, two-armed, randomized-controlled, rater-blind clinical trial with the aim to investigate whether hypnotherapy Treatment (HT) is not inferior to Cognitive Behavioral Treatment (CBT). A total of 152 patients with major depression were randomized to either CBT or HT. The CBT focused on cognitive control processes while HT on emotional activation and reinforcement of inner resources and the development of positive solution imagery. This study revealed that hypnosis is just as effective emotional control strategy as CBT, although it may use alternative neural pathways.

\section{Virtual/augmented reality hypnosis}

Virtual reality hypnosis (VRH) is a new and promising technology that uses a head mounted display to create a controlled virtual environment adapted to various techniques required in a hypnotherapeutic program [33-34]. Virtual reality hypnosis captures attention at the present moment and eliminates distracting stimuli, so it can be beneficial for those with attention deficits. VRH is also effective for people with difficulties in mental imagery replacing many stimuli that the patients struggle to imagine via verbal cueing. In addition, it holds great promise for those patients with hearing impairments [34].

Children with autism are unlikely subjects to face-to-face hypnosis due to inattentiveness and inability to receive verbal cues. Virtual technologies provide sensory stimuli in a controlled virtual environment. Austin et al. [33] conducted a feasibility study to uncover the strengths and the weaknesses of virtual reality hypnosis as an intervention tool in two boys 14 and 15 years old with severe autism. The results showed that this technique relaxed and gained patients' attention, but the procedure had no effects on autistic symptoms. However, more research is required to determine the factors that may improve VRH effectiveness in autism.

Thompson et al. [35] conducted a randomized controlled trial to investigate the effects of hypnosis with 3-D virtual reality animation on both depth of engagement and outcome measures of mood, tiredness, and salivary cortisol. Thirty-five participants were randomized in the following interventions: self-hypnosis with VR-imagery, standard 
self-hypnosis and relaxation training. The results indicated that both hypnosis groups showed lower tiredness ratings than relaxation group. However, VRH surpassed, since it helped participants with low absorption to achieve significant higher engagement which translates to psychological immersion, freedom from distraction and improved concentration. There was no significant effect on cortisol.

The combination of virtual reality with hypnosis might be an effective tool in treating phobias, avoidance, panic and generally the feeling of losing self-control. Hirsch et al. [36] presented a case study of a patient with a refractory to traditional methods phobia using virtual reality exposure therapy (VRET) combined with hypnosis. After gradual exposure and desensitization, the patient was confident and capable of coping with his fears. Virtual reality helped him reframe actual situations as being "simulation" of the virtual reality exposure. Similarly, Kraft et al. [37] in their case study achieved complete recovery for a patient with phobia through systematic desensitization with VRH.

The sense of presence is a fundamental component of virtual reality experience as well as hypnosis. Opris et al. [38] exposed 60 participants to a virtual environment, while only 30 subjects in the experimental group received hypnotic induction and suggestions. According to the questionnaire that measured presence, the experimental group experiences the sense of presence at a higher degree.

People with disabilities often experience the subjective sense of pain. Patterson et al. [39] used a case study approach in order to explore the use of hypnosis through a 3-dimensional, immersive, computer-generated virtual reality world as a means of pain and anxiety management. The post-hypnotic suggestions within the virtual environment decreased pain and anxiety by nearly 40 percent. Similarly, Teeley et al. [40] achieved to reduce the feeling of pain from $70 \%$ to $30 \%$ in three patients using VRH. VRH captured subjects' attention facilitating response to hypnosis, while, at the same time, distracting their attention from painful procedures. Patterson at al. [41], in a randomized control trial, found that VR with post-hypnotic suggestion brought better results in comparison with virtual reality distraction. Suggestions asking patients to forget pain, to recall positive experiences, to imagine a better future, to feel emotional calm seem to have an impact on pain management. Patterson et al. [42] concluded that VRH was a more effective strategy rather than hypnotic analgesia alone during thermal pain. According to the scoping review conducted by Rousseaux et al. [6], VRH in addition to reduction in pain intensity and unpleasantness, also decreases time spent in stressful thinking about pain.

Augmented reality technology (ARH) is up for playing an important role in therapeutic interventions and specifically in clinical hypnosis. Zhao et al. [43] investigated the effectiveness of an augmented reality hypnosis on psychological stress in comparison with an ordinary hypnosis. Forty-nine students with anxiety were assigned to the test or control group. The experimental group was instructed with the aid of an AR hypnotic experiment interface. The results revealed that AR hypnosis was more effective than ordinary hypnosis in terms of anxiety. AR hypnosis surpassed ordinary hypnosis possibly because it can emerge more bodily senses, expand physical and mental world, enhance subjects' imagery and memory. AR hypnosis also provides many other opportunities for patients as well as counselors to achieve the optimal outcome. 


\section{$4 \quad$ Discussion and conclusions}

The current review paper is aimed at investigating the impact of clinical hypnosis on people with physical, emotional, behavioral, or learning disabilities. Overall, the findings confirm the hypothesis that hypnosis has a positive impact on a wide range of functions, thus making it an effective intervention strategy (Table 1).

Table 1. The impact of clinical hypnosis in special education domains

\begin{tabular}{|c|c|}
\hline ADHD & $\begin{array}{l}\text { - Reduced inattentiveness \& hyperactivity } \\
\text { - Increased positivity } \\
\text { - Decreased reaction time }\end{array}$ \\
\hline ASD & $\begin{array}{l}\text { - Joint attention } \\
\text { - Emotional receptivity \& comprehension } \\
\text { - Improved motor behaviour } \\
\text { - Improved self-control \& social engagement } \\
\text { - Improved observation \& recognition skills }\end{array}$ \\
\hline DYSLEXIA & $\begin{array}{l}\text { - Better working memory } \\
\text { - } \text { Visuo-spatial mental imagery skills }\end{array}$ \\
\hline $\begin{array}{l}\text { PHYSICAL } \\
\text { IMPAIRMENTS }\end{array}$ & $\begin{array}{l}\text { - } \text { Motor imagery } \\
\text { - } \text { Motor control } \\
\text { - Implicit learning }\end{array}$ \\
\hline DOWN & - Improved retrieval of information \\
\hline $\begin{array}{l}\text { LANGUAGE } \\
\text { DISORDERS }\end{array}$ & $\begin{array}{l}\text { - Fluency/regulation of speech } \\
\text { - Reading \& listening comprehension } \\
\text { - Speed } \\
\text { - Resilience to failure }\end{array}$ \\
\hline ANXIETY & $\begin{array}{l}\text { - Improved inhibiting the reaction of fear } \\
\text { - Decreased activation of the anxiery circuity (i.e. amygdala) }\end{array}$ \\
\hline DEPRESSION & $\begin{array}{l}\text { - Disengagement from negative self-hypnosis } \\
\text { - Creating new pathways of positivity \& happiness }\end{array}$ \\
\hline GIFTEDNESS & $\begin{array}{l}\text { - Creativity \& Problem Solving } \\
\text { - Intrinsic motivation \& reward } \\
\text { - Flow, peak performance \& enjoyment } \\
\text { - Expansion of perception, prediction \& unconditional love }\end{array}$ \\
\hline
\end{tabular}

Specifically, it has been found that hypnosis eliminates symptoms of inattentiveness, distraction, and hyperactivity. It makes participants' reactions faster and more accurate. It cultivates many aspects of executive functions and boosts emotional regulation [7-8].

In the case of the Autism Spectrum Disorder, the findings suggest improvements in joint attention, emotional receptivity and comprehension, motor control and social engagement [9]. There are also indications that hypnosis enhances observation and recognition skills and benefits overall self-control processes in real situations [10].

Hypnosis' tools are considered attention and mental imagery and possibly for that reason it is associated with better working memory capacity and visuospatial skills. It also facilitates retrieval of information [13-14]. There is also evidence about hypnosis' positive impact on higher motor control processes [15,17]. In regards with language difficulties, hypnosis regulates speech and fluency [20-22], it boosts reading and listening speed and comprehension. 
The findings also support the idea that clinical hypnosis creates the conditions for peak performance by expanding perception, achieving flow state, and mobilizing higher mental abilities such as those of creativity, problem solving and prediction. At the same time, trance gives rise to superior inner forces namely intrinsic motivation and reward. In some cases, it might help one to transcend boundaries and self-imposed limits experiencing unconditional love [23-25].

A significant percentage of disabled could face symptoms of anxiety or depression. Clinical hypnosis suspends the circuitry of fear allowing disengagement from the negative self-hypnosis pathways possibly derived from learned weaknesses namely rumination and distorted memories while opening new avenues of self-governance [30-32].

Virtual reality and hypnosis have the significant potential for a synergistic effect with attentional and imagery mechanisms as a common denominator [34]. VRH facilitates hypnotic procedures by capturing and enhancing attention and concentration on the present moment [38], minimizing distraction, and boosting imagery especially in the case of low imagination individuals [34]. It was found that this is an effective strategy for disorders refractory to traditional hypnotic methods [36].

Augmented reality hypnosis is a promising therapy technology in cases of stress and anxiety. It was found that its effectiveness surpasses the ordinary therapy. In addition, it provides advantages for patients as well as counselors improving the probability of success through controlled multi-sensory stimulation and sensitivity tests.

Clinical hypnosis, as part of educational psychology, could be applied easily, rapidly at a minimal cost in educational institutions by a specialized and experienced hypnotherapists or trained educators as an intervention tool accordingly with students' existing strengths and weaknesses. Apart from individual interventions, group hypnosis intervention programs could be implemented since they are widely accepted and very successful in cultivating self-management skills [44]. Hypnosis could also enhance teachers' skills. Ghasemi et al. [45] observed that hypnosis had a positive impact on novice and expert teachers enabling them to control their cognition as well as emotions, to recognize students' emotions, to alter fixed beliefs and cognitions about teaching, to adopt positive attitudes.

Virtual reality hypnosis is low cost, easy to use and provides various opportunities to enrich the hypnotherapeutic program with various techniques [33]. VRH eliminates the need for the physical presence of a clinician, so it could be used in educational settings [34].

According to Drigas et al. human intelligence unfolds as knowledge being organized and transformed through metacognition in superior levels of consciousness [46-48]. Ascending to the higher levels of human consciousness is unachievable without the previous liberation from the "chains of slavery" that shape human's perception, preventing hidden potential to break the barriers of sub-consciousness and reach the light of consciousness. The "chains of slavery" are all those factors (such as dysfunctional or overlearned schemas, distorted beliefs, defence mechanisms, conflicting thoughts, unwanted automatisms) that slow down metacognitive evolution and form the background of various disorders. However, subconsciousness is still holding the keys for unlocking one's inner liberating forces towards autonomy and inclusion [49]. Metacognition and well-being — although it may seem contradictory_are largely dependent 
on non-conscious operations. Most of our conscious decisions are guided by forces that possibly we are unaware. Let us consider autonomic nervous system, hormonal as well as stress and reward system. All these systems, guided mainly from subconsciousness processes, determine human's future in terms of health, intelligence, metacognition [50]. According to Drigas et al. clinical hypnosis gives access to the unexplored sides of cognition where the healing powers of sub-consciousness restore homeostasis and open new avenues of metacognitive development [51]. Indeed, in this review study it was confirmed that clinical hypnosis can help people with disabilities to re-establish homeostasis, activate dormant abilities and develop metacognitive skills through the non-verbal pathways of effortless self-regulation.

We are on the eve of a new philosophy in special education, open to ground-breaking intervention strategies that recognize the fundamental role of subconsciousness in the treatment of learning and other disabilities. Clinical hypnosis seems to be a promising strategy with high potential to be applied in the educational settings.

Although this paper was not exhaustive it has demonstrated significant evidence regarding the role of clinical hypnosis as a significant intervention strategy in special education. Further well-designed experiments including randomized control trials, need to assess the effectiveness of clinical hypnosis in students' population as well as in large groups of people with disabilities and learning difficulties. Virtual/augmented reality hypnosis provided promising results, but further research is needed to confirm its effectiveness in various disabilities including learning disabilities.

\section{$5 \quad$ References}

[1] Shrestha, M., Lautenschleger, J., \& Soares, N. (2020). Non-pharmacologic management of attention-deficit/hyperactivity disorder in children and adolescents: a review. Translational Pediatrics, 9(Suppl 1), S114. https://doi.org/10.21037/tp.2019.10.01

[2] Sawni, A., \& Breuner, C. C. (2017). Clinical hypnosis, an effective mind-body modality for adolescents with behavioral and physical complaints. Children, 4(4), 19. https://doi. org/10.3390/children4040019

[3] Mendoza, M. E., \& Capafons, A. (2009). Efficacy of clinical hypnosis: a summary of its empirical evidence. Papeles del Psicólogo.

[4] Elkins, G. R., Barabasz, A. F., Council, J. R., \& Spiegel, D. (2015). Advancing research and practice: the revised APA division 30 definition of hypnosis. American Journal of Clinical Hypnosis, 57(4), 378-385. https://doi.org/10.1080/00029157.2015.1011465

[5] Lifshitz, M., Cusumano, E. P., \& Raz, A. (2013). Hypnosis as neurophenomenology. Frontiers in Human Neuroscience, 7, 469. https://doi.org/10.3389/fnhum.2013.00469

[6] Rousseaux, F., Bicego, A., Ledoux, D., Massion, P., Nyssen, A. S., Faymonville, M. E., ... Vanhaudenhuyse, A. (2020). Hypnosis associated with 3D immersive virtual reality technology in the management of pain: a review of the literature. Journal of Pain Research, 13, 1129. https://doi.org/10.2147/JPR.S231737

[7] Hiltunen, S., Virta, M. A. A. R. I. T., Salakari, A. N. I. T. A., Antila, M. E. R. V. I., Chydenius, E., Kaski, M., \& Partinen, M. (2014). Better long-term outcome for hypnotherapy than for CBT in adults with ADHD: results of a six-month follow-up. Contemporary Hypnosis \& Integrative Therapy, 30(3), 118-134.

[8] Virta, M., Hiltunen, S., Mattsson, M., \& Kallio, S. (2015). The impact of hypnotic suggestions on reaction times in continuous performance test in adults with ADHD and healthy controls. Plos One, 10(5), e0126497. https://doi.org/10.1371/journal.pone.0126497 
[9] Sugarman, L. I., Garrison, B. L., \& Williford, K. L. (2013). Symptoms as solutions: hypnosis and biofeedback for autonomic regulation in autism spectrum disorders. American Journal of Clinical Hypnosis, 56(2), 152-173. https://doi.org/10.1080/00029157.2013.768197

[10] Gardner, G. G., \& Tarnow, J. D. (1980). Adjunctive hypnotherapy with an autistic boy. American Journal of Clinical Hypnosis, 22(3), 173-179. https://doi.org/10.1080/00029157. 1980.10403222

[11] Giovagnoli, G., Vicari, S., Tomassetti, S., \& Menghini, D. (2016). The role of visual-spatial abilities in dyslexia: age differences in children's reading? Frontiers in Psychology, 7, 1997. https://doi.org/10.3389/fpsyg.2016.01997

[12] Szucs, D., Devine, A., Soltesz, F., Nobes, A., \& Gabriel, F. (2013). Developmental dyscalculia is related to visuo-spatial memory and inhibition impairment. Cortex, 49(10), 2674-2688. https://doi.org/10.1016/j.cortex.2013.06.007

[13] Incognito, O., Menardo, E., Di Gruttola, F., Tomaiuolo, F., Sebastiani,L., \& Santarcangelo, E. L. (2019). Visuospatial imagery in healthy individuals with different hypnotizability levels. Neuroscience Letters, 690, 158-161. https://doi.org/10.1016/j.neulet.2018.10.039

[14] Lindeløv, J. K., Overgaard, R., \& Overgaard, M. (2017). Improving working memory performance in brain-injured patients using hypnotic suggestion. Brain, 140(4), 1100-1106. https://doi.org/10.1093/brain/awx001

[15] Müller, K., Bacht, K., Schramm, S., \& Seitz, R. J. (2012). The facilitating effect of clinical hypnosis on motor imagery: an fMRI study. Behavioural Brain Research, 231(1), 164-169. https://doi.org/10.1016/j.bbr.2012.03.013

[16] Gravitz, M. A. (1981). Non-verbal hypnotic techniques in a centrally deaf brain-damaged patient. International Journal of Clinical and Experimental Hypnosis, 29(2), 110-116. https://doi.org/10.1080/00207148108409151

[17] Flamand-Roze, C., Célestin-Lhopiteau, I., \& Roze, E. (2016). Hypnosis and movement disorders: state of the art and perspectives. Revue Neurologique, 172(8-9), 530-536. https:// doi.org/10.1016/j.neurol.2016.07.008

[18] Garitte, C., Gay, M. C., Cuisinier, F., \& Celeste, B. (2009). Hypnotic susceptibility in children with down's syndrome. Contemporary Hypnosis, 26(2), 111-120. https://doi. org $/ 10.1002 /$ ch.374

[19] Iglesias, A., \& Iglesias1, A. (2014). Pediatric emotional dysregulation and behavioral disruptiveness treated with hypnosis: a time-series design. International Journal of Clinical and Experimental Hypnosis, 62(1), 70-83. https://doi.org/10.1080/00207144.2013.841480

[20] Kaya, Y., \& Alladin, A. (2012). Hypnotically assisted diaphragmatic exercises in the treatment of stuttering: a preliminary investigation. International Journal of Clinical and Experimental Hypnosis, 60(2), 175-205. https://doi.org/10.1080/00207144.2012.648063

[21] Sehan, Z., Harun, M., \& Ahmad, I. (2017). The effects of light trance and post-hypnotic suggestions towards the university students' reading comprehension improvement. Sleep and Hypnosis (Online), 19(4), 78-82. https://doi.org/10.5350/Sleep.Hypn.2016.18.0125

[22] Wark, D. M. (2011). Traditional and alert hypnosis for education: a literature review. American Journal of Clinical Hypnosis, 54(2), 96-106. https://doi.org/10.1080/00029157.2 $\underline{011.605481}$

[23] Bowers, P. (1979). Hypnosis and creativity: the search for the missing link. Journal of Abnormal Psychology, 88(5), 564. https://doi.org/10.1037/0021-843X.88.5.564

[24] Gur, R. C., \& Reyher, J. (1976). Enhancement of creativity via free-imagery and hypnosis. American Journal of Clinical Hypnosis, 18(4), 237-249. https://doi.org/10.1080/00029157. 1976.10403806

[25] Bowers, J., Na, H., \& Elkins, G. (2018). Flow and hypnotizability in a college student population. International Journal of Clinical and Experimental Hypnosis, 66(3), 331-342. https://doi.org/10.1080/00207144.2018.1468159 
[26] Pates, J., \& Palmi, J. (2002). The effects of hypnosis on flow states and performance. Journal of Excellence, 6, 48-62.

[27] Mossbridge, J. A., Nisam, M., \& Crabtree, A. (2020). Can hypnotic suggestion induce feelings of unconditional love and supernormal performance? Spirituality in Clinical Practice. https://doi.org/10.1037/scp0000239

[28] Valentine, K. E., Milling, L. S., Clark, L. J., \& Moriarty, C. L. (2019). The efficacy of hypnosis as a treatment for anxiety: a meta-analysis. International Journal of Clinical and Experimental Hypnosis, 67(3), 336-363. https://doi.org/10.1080/00207144.2019.1613863

[29] Byron, D. (2002). The use of hypnosis to help an anxious student with a social communication disorder to attend school. Contemporary Hypnosis, 19(3), 125-132. https://doi. org/10.1002/ch.249

[30] Halsband, U., \& Wolf, T. G. (2015). Functional changes in brain activity after hypnosis in patients with dental phobia. Journal of Physiology-Paris, 109(4-6), 131-142. https://doi. org/10.1016/j.jphysparis.2016.10.001

[31] Alladin, A., \& Alibhai, A. (2007). Cognitive hypnotherapy for depression: an empirical investigation. International Journal of Clinical and Experimental Hypnosis, 55(2), 147-166. https://doi.org/10.1080/00207140601177897

[32] Fuhr, K., Meisner, C., Broch, A., Cyrny, B., Hinkel, J., Jaberg, J., ... Batra, A. (2021). Efficacy of hypnotherapy compared to cognitive behavioral therapy for mild to moderate depression-Results of a randomized controlled rater-blind clinical trial. Journal of Affective Disorders, 286, 166-173. https://doi.org/10.1016/j.jad.2021.02.069

[33] Austin, D. W., Abbott, J. A. M., \& Carbis, C. (2008). The use of virtual reality hypnosis with two cases of autism spectrum disorder: a feasibility study. Contemporary Hypnosis, 25(2), 102-109. https://doi.org/10.1002/ch.349

[34] Askay, S. W., Patterson, D. R., \& Sharar, S. R. (2009). Virtual reality hypnosis. Contemporary Hypnosis, 26(1), 40-47. https://doi.org/10.1002/ch.371

[35] Thompson, T., Steffert, T., Steed, A., \& Gruzelier, J. (2010). A randomized controlled trial of the effects of hypnosis with 3-D virtual reality animation on tiredness, mood, and salivary cortisol. International Journal of Clinical and Experimental Hypnosis, 59(1), 122-142. https://doi.org/10.1080/00207144.2011.522917

[36] Hirsch, J. A. (2012). Virtual reality exposure therapy and hypnosis for flying phobia in a treatment-resistant patient: a case report. American Journal of Clinical Hypnosis, 55(2), 168-173. https://doi.org/10.1080/00029157.2011.639587

[37] Kraft, T., \& Kraft, D. (2004). Creating a virtual reality in hypnosis: a case of driving phobia. Contemporary Hypnosis, 21(2), 79-85. https://doi.org/10.1002/ch.293

[38] Opris, D., Enea, V., Pop, A., \& Dafinoiu, I. (2011). Hypnotic suggestions effect on sense of presence in virtual reality. A brief report. Erdélyi Pszichológiai Szemle, 12(1), 13-22.

[39] Patterson, D. R., Tininenko, J. R., Schmidt, A. E., \& Sharar, S. R. (2004). Virtual reality hypnosis: a case report. International Journal of Clinical and Experimental Hypnosis, 52(1), 27-38. https://doi.org/10.1076/iceh.52.1.27.23925

[40] Teeley, A. M., Soltani, M., Wiechman, S. A., Jensen, M. P., Sharar, S. R., \& Patterson, D. R. (2012). Virtual reality hypnosis pain control in the treatment of multiple fractures: a case series. American Journal of Clinical Hypnosis, 54(3), 184-194. https://doi.org/10.1080/000 29157.2011.619593

[41] Patterson, D. R., Jensen, M. P., Wiechman, S. A., \& Sharar, S. R. (2010). Virtual reality hypnosis for pain associated with recovery from physical trauma. International Journal of Clinical and Experimental Hypnosis, 58(3), 288-300. https://doi.org/10.1080/00207141003760595 
[42] Patterson, D. R., Hoffman, H. G., Chambers, G., Bennetts, D., Hunner, H. H., Wiechman, S. A., \& Jensen, M. P. (2021). Hypnotic enhancement of virtual reality distraction analgesia during thermal pain: a randomized trial. International Journal of Clinical and Experimental Hypnosis, 69(2), 225-245. https://doi.org/10.1080/00207144.2021.1882259

[43] Zhao, X., You, X., Shi, C., \& Gan, S. (2015). Hypnosis therapy using augmented reality technology: treatment for psychological stress and anxiety. Behaviour \& Information Technology, 34(6), 646-653. https://doi.org/10.1080/0144929X.2015.1022223

[44] Fisch, S., Binting, S., Roll, S., Cree, M., Brinkhaus, B., \& Teut, M. (2020). Group hypnosis for stress reduction-a feasibility study. International Journal of Clinical and Experimental Hypnosis, 68(4), 493-510. https://doi.org/10.1080/00207144.2020.1781537

[45] Ghasemi, F. (2019). Incorporating hypnotic suggestion into teacher education programs: emotional and cognitive implications for teachers. Australian Journal of Applied Linguistics, 2(3), 83-103. https://doi.org/10.29140/ajal.v2n3.174

[46] Drigas, A. S., \& Pappas, M. A. (2017). The consciousness-intelligence-knowledge pyramid: an $8 \times 8$ layer model. International Journal of Recent Contributions from Engineering, Science \& IT (iJES), 5(3), 14-25. https://doi.org/10.3991/ijes.v5i3.7680

[47] Drigas, A., \& Mitsea, E. (2020). The triangle of spiritual intelligence, metacognition and consciousness. International Journal of Recent Contributions from Engineering, Science \& IT (iJES), 8(1), 4-23. https://doi.org/10.3991/ijes.v8i1.12503

[48] Drigas, A., \& Mitsea, E. (2020). The 8 pillars of metacognition. International Journal of Emerging Technologies in Learning (iJET), 15(21), 162-178. https://doi.org/10.3991/ijet. v15i21.14907

[49] Drigas, A. \& Mitsea, E. (2021). 8 Pillars x 8 Layers model of metacognition. Educational strategies, exercises \& trainings. International Journal of Online \& Biomedical Engineering. 17(8), 115-134. https://doi.org/10.3991/ijoe.v17i08.23563

[50] Drigas, A., \& Mitsea, E. (2021). Metacognition, stress-relaxation balance \& related hormones. International Journal of Recent Contributions from Engineering, Science \& IT (iJES), 9(1), 4-16. https://doi.org/10.3991/ijes.v9i1.19623

[51] Drigas, A. \& Mitsea, E. (IJOE submitted). Clinical Hypnosis \& VR, Subconscious Restructuring- Brain Rewiring \& the Entanglement with the 8 Pillars of Metacognition $\times 8$ Layers of Consciousness $\times 8$ Intelligences.

\section{Authors}

Athanasios Drigas is a Research Director at IIT-N.C.S.R. 'Demokritos', Institute of Informatics and Telecommunications - Net Media Lab \& Mind - Brain R \& D, Agia Paraskevi, 15310, Athens, Greece (dr@iit.demokritos.gr).

Charalabos Skianis is Vice Rector Finance, Planning \& Development at University of the Aegean and a Professor in the Department of Information and Communication Systems Engineering at the University of the Aegean, Greece (cskianis@,aegean.gr).

Eleni Mitsea is a Phd Candidate in the Department of Information \& Communication Systems Engineering at the Aegean University in collaboration with the Institute of Informatics and Telecommunications Net Media Lab, Athens, Greece (e.mitsea@gmail.com)

Article submitted 2021-08-10. Resubmitted 2021-10-15. Final acceptance 2021-10-15. Final version published as submitted by the authors. 